\title{
Ueber die Anwendbarkeit des periodischen Gesetzes bei den Ceritmetallen; eine Erwiderung ${ }^{*}$ ) von $D$. Mendelejeff.
}

(Eingelaufen den 19. April 1873.)

Die periodische Abhängigkeit der Atomgewichte der Elemente von deren Eigenschaften mufs auch bei den seltenen Metallen angewandt werden, um denselben entsprechenden Platz unter den übrigen Elementen anzuweisen und zugleich die allgemeine Gültigkeit des Gesetzes prüfen zu können. In einer vorhergehenden Abhandlung **) habe ich üher eine derartige Anwendung des neuen Gesetzes berichtet. Wäre das Gesetz kein allgemeines und böte es nicht den Schlüssel zur Erledigung der Fragen über Elemente, so wäre ich dabei auf Hindernisse gestofsen und hätte Ausnahmen (corps à sérier) zulassen müssen, was bei strengen Naturgesetzen nicht sein darf. Doch hat nichts Derartiges stattgefunden. Im Gegentheil, bei allen Elementen hat das Gesetz Anwendung gefunden, was einen überzeugenden Beweis von dessen Richtigkeit liefert. Um zu einer solchen Uebereinstimmung zu gelangen, mufsten zwar bei einigen Elementen die bisher angenommenen Atomgewichte abgeändert werden; doch war eine Abänderung bei keinem von den genügend bekannten Elementen, für welche die Atomgewichte als genau festgestellt anzunehmen sind, von Nöthen; sie betraf, was sehr wichtig ist,

*) Siehe Rammelsberg, Berichte der deutschen chemischen Gesellschaft $6,84$.

**) Eine ausführlich $\theta$ Abbandlung über diesen Gegenstand ist in diesen Annalen Suppl. 8, 133 zu finden. Vordem sind dieselben Begriffe in mebreren Abhandlungen im Russ. Chem. Journal und in meinen nGrundzügen der Chemie" entwickelt worden. 
blofs einige wenig untersuchte Elemente, bezüglich welcher das Gesetz bestimmte Antwort auf die zu erledigenden Fragen gegeben hat. Die durch das periodische Gesetz hervorgerufenen Aenderungen an den Atomgewichten waren um so eher zulässig, als bei Feststellung der üblichen Atomgewichte vieler seltenen Elemente keine genügenden Beweisgründe vorhanden gewesen waren. So mufsten im Sinne des periodischen Gesetzes die Atomgewichte von In, Ur, Th, Ce, La, $\mathrm{Di}, \mathrm{Yt}, \mathrm{Er}$ abgeändert werden. Das neue Atomgewicht von In fand eine weitere Bestätigung in der von Bunsen und mir bestimmten specifischen Wärme. Für Th ist schon früher (Delafontaine, Chydenius) die aus dem periodischen Gesetze folgende Oxydformel $\mathrm{ThO}^{2}$ vorgeschlagen worden. Dem Uranoxyd habe ich die Formel $\mathrm{UrO}^{3}$ gegeben; unlängst hat Rammelsberg bezüglich des Urans meiner Beweisführung beigestimmt (Berichte der deutschen chemischen Geselischaft 5, 1003). Gegenwärtig (Berichte der deutschen chemischen Gesellschaft $\mathbf{6}, 84$ ) spricht sich derselbe Kenner seltener Metalle gegen die von mir vorgeschlagenen Abänderungen an den Atomgewichten von Cerium *), Didym und Lanthan aus. Da ich R a mmelsberg's Deutung der bezüglichen Thatsachen für ungenügend und zum Theil letzteren nicht entsprechend halte, da derselbe auf kein anderes Mittel als das von mir befolgte Verfahren, um die das Cerium betreffenden Thatsachen mit dem periodischen Gesetz in Uebereinstimmung zu bringen, hinweist, und da ich das periodische Gesetz für wahr halte, so erwidere ich Rammelsberg, indem ich alle seine Beweise und Bemerkungen ohne Ausnahme einer näheren Betrachtung unterwerfe **).

*) Bull. de l'Acad. de St. Pét. 1870, 445 und diese Annalen a. a. O.

**) Ich erlaube mir zu bemerken, dafs H. Rose kein abgeändertes Atomgewicht vorgeschlagen hat. In der von Rammelsberg 
Rammelsberg bespricht zur Widerlegung des von mir angenommenen Atomgewichts von Cerium (138) die Zusammensetzung der Ceroxydsalze. Seiner Meinung nach mufs man beim neuen Atomgewicht aufser dem Oxydul $\mathrm{Ce}^{2} 0^{3}$ und dem Oxyd $\mathrm{CeO}^{2}$, nicht blofs ein intermediäres Oxyd $\mathrm{Ce}^{5} \mathrm{O}^{3}=\mathrm{Ce}^{2} \mathrm{O}^{3}, 3 \mathrm{CeO}^{2}$ (d. h. $\mathrm{Ce}^{5} \mathrm{O}^{6}$, wenn $\mathrm{Ce}=92$ ), sondern auch eine höhere Oxydationsstufe $\mathrm{Ce}^{10} 0^{21}$ (früher $\mathrm{Ce}^{5} 0^{7}$ ) annehmen, was die Möglichkeit meiner Annahme, nach welcher $\mathrm{Ce}$ in die IV. Gruppe, mit der höchsten Oxydationsstufe $\mathrm{RO}^{2}$ gehört, ausschliefst. $\mathrm{Zu}$ solchen Schlüssen gelangt Rammelsberg, indem er die Analysen zweier von ihm untersuchten Salze discutirt (Pogg. Ann. 108, 45 und 58). Streng genommen genügen diese Thatsachen nicht; doch hoffe ich selbst an den zwei angeführten Beispielen nachzuweisen, dafs Rammelsberg's Data besser durch andere und einfachere Formeln, welche vollkommen mit dem abgeänderten Atomgewicht übereinstimmen, ausgedrückt werden können. Was die übrigen Cersalze anbelangt, so widersprechen dieselben auch nach Rammelsberg nicht meinen Ansichten. Das Oxyd $\mathrm{Ce}^{5} 0^{3}$ nimmt $\mathrm{R}$ a mmelsberg in dem braunrothen (sechsgliedrigen), gut krystallisirenden schwefelsauren Salze an, welches sich beim Verdunsten einer Lösung von Ceroxyd $\mathrm{Ce}^{2}$ in überschüssiger Schwefelsäure ausscheidet. Bezüglich dieses Salzes ist zu bemerken, dafs es als Salz eines schwach basischen Oxyds, ähnlich den Wismuthoxydsalzen u. s. w., von Wasser in Ueberschufs besonders beim Erwärmen zer-

citirten Fufsnote (H. Rose, analyt. Chemie, 6. Aufl., I, 219) ist nicht vom Atomgewicht des Ceriums die Rede, sondern es wird die bis anf Marignac (1848) üblich gewesene Bezeichnung der höchsten Oxydationsstufe $\left(\mathrm{Ce}^{3} \mathrm{O}^{4}\right.$ wenn $\mathrm{Ce}=92$ oder $\mathrm{CeO}^{2}$ wenn $\mathrm{Ce}=138$ ) als Ceroxyd, nicht als Ceroxyduloxyd, wie $\mathrm{Ram}$ melsberg dieselbe noch gegenwärtig nennt, wieder aufgenommen. Die Oxydationsstufe $\mathrm{CeO}^{2}(\mathrm{Ce}=138)$ nenne auch ich Oxyd. 
setzt wird, wobei ein basisches Salz entsteht; bei gelindem Erwärmen bildet sich theilweise Oxydulsalz, wie aus dem Gehalt an Ceroxydul in dem aus Ceroxyd bereiteten Salz zu ersehen ist. Folglich läfst sich das erwähnte Salz nicht vollkommen rein darstellen, was ferner bestätigt wird : durch die mangelnde Uebereinstimmung in den analytischen Daten von Herrmann (Journal für praktische Chemie 30, 184 und 92, 113), Zschiesche (daselbst 10\%, 82 bis 86) und Rammelsberg; dadurch, dafs Rammelsberg's Formel weniger $\mathrm{SO}^{3}$ (33,9 pC.) verlangt, als die Analyse ergeben hat $(36,4)$, was er durch die Unmöglichkeit Spuren überschüssiger Schwefelsäure zu entfernen erklärt; schliefslich durch die diesem Salze von verschiedenen Forschern zu verschiedenen Zeiten gegebenen nicht übereinstimmenden Formeln; nämlich

bei Annahme des Oxyds als ( $\mathrm{Ce}=92)$

$\begin{array}{cc}\mathrm{Ce}^{2} \mathrm{O}^{3} & \mathrm{Ce}^{5} \mathrm{O}^{6} \\ (\mathrm{Herrmann}, 1843) & (\mathrm{Rammelsberg}, 1859) \\ \mathrm{Ce}^{2}\left(\mathrm{SO}^{4}\right)^{3}, 9 \mathrm{H}^{2} \mathrm{O} & 3 \mathrm{Ce}\left(\mathrm{SO}^{4}\right) \\ & \left.-\mathrm{Ce}^{2}\left(\mathrm{SO}^{4}\right)^{3}\right\} 18 \mathrm{H}^{2} \mathrm{O}\end{array}$

$\mathrm{Ce}^{6} \mathrm{O}^{7}$

$\mathrm{Ce}^{7} \mathrm{O}^{8}$

$\mathrm{Ce}^{5} \mathrm{O}^{6}$

(Herrmann, 1865) Zschiesche, 1869) (Rammelsberg, 1873)

\begin{tabular}{|c|c|c|c|c|}
\hline $\left.\begin{array}{l}4 \mathrm{Ce}\left(\mathrm{SO}^{4}\right) \\
\mathrm{Ce}^{2}\left(\mathrm{SO}^{4}\right)^{3} \\
2 \mathrm{H}^{2}\left(\mathrm{SO}^{4}\right)\end{array}\right\}$ & $25 \mathrm{H}^{2} \mathrm{O}$ & $\left.\begin{array}{l}5 \mathrm{Ce}\left(\mathrm{SO}^{4}\right) \\
\mathrm{Ce}^{2}\left(\mathrm{SO}^{4}\right)^{3} \\
\mathrm{H}^{2}\left(\mathrm{SO}^{4}\right)\end{array}\right\}$ & $26 \mathrm{H}^{2} \mathrm{O}$ & $\left.\begin{array}{l}3 \mathrm{Ce}\left(\mathrm{SO}^{4}\right) \\
\mathrm{Ce}^{2}\left(\mathrm{SO}^{4}\right)^{3}\end{array}\right\} 21 \mathrm{H}^{2} \mathrm{O}$ \\
\hline
\end{tabular}

Nur die erste der angeführten Formeln ist bei Annahme des neuen Atomgewichts unmöglich ( $\mathrm{Ce}=138)$, da sie einem höheren $\mathrm{Oxyde}$, als $\mathrm{CeO}^{2}$ entspricht; die übrigen sind wenn auch möglich, doch ihrer Complicirtheit halber wenig wahrscheinlich, mag man $\mathrm{Ce}=92$ oder 138 annehmen. Um die richtige Zusammensetzung des Salzes aufzufinden, will ich blofs Rammelsberg's eigene Data in Betracht ziehen; aufserdem sind dieselben vollständiger und stimmen besser untereinander, als die Angaben von Herrmann und Zschiesche. Dabei mufs man dem Wunsche, eine genaue 
allgemeine Formel aufzufinden entsagen, weil der Krystallwassergehalt nicht bestimmt worden ist, und in dem Salze, wie oben erwähnt, voraussetzlich überschüssige freie Schwefelsäure enthalten sein mufs. Uebrigens handelt es sich nicht darum, sondern um die Zusammensetzung des im Salze enthaltenen Ceriumoxyds, wozu wir bei Rammelsberg genügend Data vorfinden. Er hat nämlich im Jahre 1859 je eilf Bestimmungen von $\mathrm{Ce}^{2}$ und des sich beim Uebergang von $2 \mathrm{CeO}^{2}$ in das Oxydul $\mathrm{Ce}^{2} \mathrm{O}^{3}$ entwickelnden Sauerstoffs ausgeführt, sowie im Jahre 1873 sechs Bestimmungen von $\mathrm{Ce}^{2}\left(\mathrm{SO}^{4}\right)^{3}$, eine von $\mathrm{CeO}^{2}$ und zwei Sauerstoffbestimmungen. Die verhältnifsmälsig gröfsten Differenzen sind beim Sauerstoff vorhanden (gefunden 0,81 bis 1,16 ). Im Mittel hat Rammelsberg 1859 gefunden $39,25 \mathrm{Ce}^{2}$ und 0,95 Sauerstoff, 1873 (nach dem schwefelsauren Salze berechnet) 40,03 $\mathrm{Ce}^{2}$ und 0,98 Sauerstoff. Folglich ist in beiden Fällen das Verhältnifs von $\mathrm{CeO}^{2}: 0=100: 2,18$; die gegenwärtig gefundenen Werthe sind, da das Salz zur Analyse noch mehr gereinigt worden war, grölser, als die von 1859. Wäre im besagten Salze alles $\mathrm{Ce}$ als $\mathrm{Ce}^{2}$ enthalten, so berechneten sich auf $100 \mathrm{Th}$. $\mathrm{CeO}^{2} 4,70 \mathrm{Th}$. Sauerstoff; gefunden ist im Mittel beinahe nur die Hälfte; folglich mufs das Salz das Oxyd $\mathrm{Ce}^{4} \mathrm{O}^{7}=\mathrm{Ce}^{2} \mathrm{O}^{3}, 2 \mathrm{CeO}^{2}$ enthalten. Letztere Annahme entspricht am Besten und Einfachsten den Analysen; denn vorausgesetzt, dafs von $40 \mathrm{pC}$. $\mathrm{Ce}^{2} 20 \mathrm{pC}$. als $\mathrm{Ce}^{2} 0^{3}$, die übrigen $20 \mathrm{pC}$. als $\mathrm{CeO}^{2}$ enthalten seien, so lassen sich $0,94 \mathrm{pC}$. oxydirenden Sauerstoffs im Salze berechnen, während $0,97 \mathrm{pC}$. Sauerstofl im Mittel $(0,81$ bis 1,16) gefunden worden sind. Folglich ist kein Grund vorhanden, der complicirteren Oxydformel von Rammelsberg $\mathrm{Ce}^{5} 0^{9}=\mathrm{Ce}^{2} 0^{3}, 3 \mathrm{CeO}^{2}$ den Vorzug vor $\mathrm{Ce}^{4} 0^{7}$ zu geben. Aufserdem entspricht letztere auch der Formel, sowie den Daten von Herrmann. Ist einmal die Zusammensetzung des $0 x y d s=\mathrm{Ce}^{2} \mathrm{O}^{3}, \mathrm{Ce}^{2} \mathrm{O}^{4}$ fest- 
gestellt, so ist auch die Zusammensetzung des Salzes, wenn man es für normal hält, in Hinsicht auf den Schwefelsäuregehalt verständlich $\mathrm{Ce}^{2} \mathrm{O}^{3}\left(\mathrm{SO}^{3}\right)^{3}, \mathrm{Ce}^{2} \mathrm{O}^{4}\left(\mathrm{SO}^{3}\right)^{4}$ *). Was den Wassergehalt anbelangt, so mufs man sich damit begnügen, dafs in den Analysen von 1859 die Differenz bis 100 25,33 ausmacht und dafs $\mathrm{Z}$ s chie s che direct 23,57 pC. Wasser gefunden hat. Diese Zahlen entsprechen am Nächsten der Formel

$\left.\begin{array}{l}\mathrm{Ce}^{2}\left(\mathrm{SO}^{4}\right)^{3} \\ \mathrm{Ce}^{2}\left(\mathrm{SO}^{4}\right)^{4}\end{array}\right\} 24 \mathrm{H}^{2} \mathrm{O}(\mathrm{Ce}=138)$, oder wenn $\mathrm{Ce}=92, \underset{\mathrm{Ce}\left(\mathrm{SO}^{4}\right)}{\left.\mathrm{Ce}^{3}\left(\mathrm{SO}^{4}\right)^{4}\right)^{24}} \mathrm{H}^{2} \mathrm{O}$.

Doch läfst sich kein strenger Vergleich der gefundenen und berechneten Werthe anstellen, da in dem analysirten Salze überschüssige freie Schwefelsäure anzunehmen ist und dasselbe überhaupt noch eines weiteren Studiums bedarf. Somit widerspricht das erwähnte Salz durchaus nicht dem von mir für Cerium vorgeschlagenen Atomgewichte; im Gegentheil, letztere Annahme führt zu einer einfacheren und wahrscheinlicheren Formel, als die von $\mathrm{Rammelsberg.}$

Das zweite Salz, welches nach $R$ a m m elsberg mit dem neuen Atomgewicht von Cerium in Widerspruch stehen soll, ist beständiger, krystallisirt in wohlausgebildeten Formen und bietet daher mehr Zuverlässigkeit was Reinheit anlangt. In Folge dessen läfst sich an diesem Salze am Besten die Richtigkeit der alten oder neuen Anschanungsweise prüfen; um so mehr, als gerade die Zusammensetzung dieses Salzes, wenn R a m melsberg's Formel die richtigo ist, meinen Ansichten widerspricht. Dasselbe wird beim Mischen einer Lösung von schwefelsaurem Ceroxyd mit $\mathrm{Am}^{2} \mathrm{SO}^{4}$ erhalten. Beim Eindampfen scheidet sich ein gelbes, krystallinisch körniges, schwerlösliches **), und ein orangerothes, gut krystallisiren-

*) Uebrigens lassen sich die Analysen, was den Schwefelsäuregehalt anbelangt, am Besten durch die Formel $\mathrm{Ce}\left(\mathrm{SO}^{4}\right)^{2}, 6 \mathrm{H}^{2} \mathrm{O}$ wiedergeben, welche 36,5 pC. $\mathrm{SO}^{3}$ verlangt (gefunden 36,4 ).

**) Nach Rammels berg $2\left(\mathrm{CeSO}^{4}\right), \mathrm{Ce}^{2}\left(\mathrm{SO}^{4}\right)^{3}, 4\left(\mathrm{Am}^{2} \mathrm{SO}^{4}\right), 4 \mathrm{H}^{2} \mathrm{O}$ 
des Salz aus; letzteres hat nach Rammelsberg folgende Zusammensetzung $(\mathrm{Ce}=92)$ :

$\left.\begin{array}{l}\begin{array}{l}\mathrm{CeSO}{ }^{4} \\ 2 \mathrm{Ce}^{2}\left(\mathrm{SO}^{4}\right)^{3}\end{array} \\ 9 \mathrm{Am}^{2} \mathrm{SO}^{4}\end{array}\right\} 12 \mathrm{H}^{2} \mathrm{O}$, d. h. wenn $\left.\mathrm{Ce}=138 \begin{array}{l}\mathrm{Ce}^{10} \mathrm{O}^{21}\left(\mathrm{SO}^{3}\right)^{21} \\ 27 \mathrm{Am}^{2} \mathrm{SO}^{4}\end{array}\right\} 36 \mathrm{H}^{2} \mathrm{O}$.

Diese Zusammensetzung ist beim neuen Atomgewicht nicht blofs der Complicirtheit halber unwahrscheinlich, sondern ganz unmöglich, weil sie zu einem noch höheren 0xyde, $\mathrm{Ce}^{111} \mathrm{O}^{21}$, als die höchst mögliche $0 x y d a t i o n s s t u f e ~ \mathrm{CeO}^{2}$ führt. Eben in Folge dieser Oxydformel spricht sich Rammelsberg gegen $\mathrm{Ce}=138$ aus. Doch wollen wir sehen, in wie weit er berechtigt ist, die Zusammensetzung seines Doppelsalzes durch die jedenfalls complicirte Formel, welche er giebt, auszudrücken.

Zuerst wollen wir die Formel des im besagten Salze enthaltenen Oxyds bestimmen; letzteres ist von Rammelsberg 1859 und 1873 untersucht worden. Erst sind im Mittel aus vier Analysen 21,84 $\mathrm{CeO}^{2}$ und aus zwei Analysen 1,28 Sauerstoff erhalten worden; gegenwärtig hat $\mathrm{R}$ a m mels berg 36,34 $\mathrm{Ce}^{2}\left(\mathrm{SO}^{4}\right)^{3}$ (resp. $21,91 \mathrm{CeO}^{2}$ ) gefunden. Im Mittel sind also

(Ce = 92). Die Mitteldifferenz zwischen den gefundenen und berechneten Werthen $= \pm 0,57$. Folgende Formel $3\left(\mathrm{Am}^{2} \mathrm{SO}^{4}\right)$, $2\left(\mathrm{CeS}^{2} \mathrm{O}^{8}\right), 3 \mathrm{H}^{2} \mathrm{O}(\mathrm{Ce}=138)$ drückt die Zusammensetzung dieses Salzes genauer aus; denn die Mitteldifferenz zwischen den berechneten und gefundenen Werthen beträgt $\pm 0,28$, und es stellt sich ein einfaches Verhältnifs dieses Salzes zum orangerothen herans. Beide anterscheiden sich von einander darin, dafs in dem einen ein $\mathrm{H}^{2} \mathrm{O}$ mehr ist, in dem anderen $\mathrm{Ce}\left(\mathrm{SO}^{4}\right)^{2}$, d. h. das orangerothe enthält im Verbältnifs zum gelben Salze doppelt so viel $\mathrm{Am}^{2} \mathrm{SO}^{4}$; das gelbe ist schwerer löslich, weil es mehr $\mathrm{Ce}\left(\mathrm{SO}^{4}\right)^{2}$ enthält. Uebrigens könnten möglicherweise letzteres wie das orangerothe Doppelsalz und das sehwefelsaure Ceroxyd nicht $3 \mathrm{H}^{2} \mathrm{O}$, sondern $4 \mathrm{H}^{2} \mathrm{O}$ enthalten. In Rammels berg's Analysen wird der Wassergehalt als aus dem Rest berechnet angefübrt $\left(5,43\right.$ pC.). Die Formel mit $4 \mathrm{H}^{2} \mathrm{O}$ verlangt $6,38 \mathrm{pC}$, mit $3 \mathrm{H}^{2} \mathrm{O}$ 4,87 pC. Wasser; folglich können blofs weitere Untersuchungen über diese Frage entscheiden. 
21,85 $\mathrm{CeO}^{2}$ gefunden, was 1,03 Sauerstoff beim Uebergang von $2 \mathrm{CeO}^{2}$ in $\mathrm{Ce}^{2} \mathrm{O}^{3}$ entspricht; da die gefundene Sauerstoffmenge 1,28 pC. betragen hat, so ist alles Cerium im Salze als $\mathrm{CeO}^{2}$ enthalten. Die Differenz zwischen dem berechneten und gefundenen Werthe $1,28-1,03=0,25 \mathrm{pC}$. liegt im Bereiche möglicher Versuchsfehler. In der That ist die Zahl 1,28 als Mittel zweier Beobachtungen erhalten worden (Pogg. Ann. 108, 59); in anderen Fällen, wo eine grölsere Anzahl Beobachtungen vorliegt, sind die Differenzen zwischen den extremen Werthen noch gröfser : 0,35 (S. 49, eilf Beobachtungen), 0,51 (S. 51, fünf Beobachtungen), 0,43 (S. 52, acht Beobachtungen), 0,52 (S. 56, acht Beobachtungen). Daraus folgt, dafs wir für das Oxyd keine andere Zusammensetzung anzunehmen berechtigt sind, als $\mathrm{CeO}^{2}$; wenn aber das Salz nur $\mathrm{CeO}^{2}$ enthält, so ist die Zusammensetzung eine sehr einfache :

$$
\left.\begin{array}{l}
\mathrm{Ce}\left(\mathrm{SO}^{4}\right)^{2} \\
3 \mathrm{Am}^{2} \mathrm{SO}^{4}
\end{array}\right\} 4 \mathrm{H}^{2} \mathrm{O} \text {. }
$$

Diese Formel *) entspricht vollkommen Rammelsb e r g 's analytischen Daten, wie folgende Zusammenstellung zeigt:

\begin{tabular}{|c|c|c|c|c|c|}
\hline & 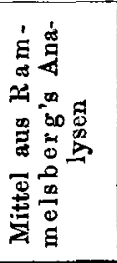 & 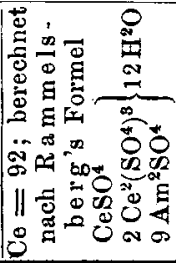 & 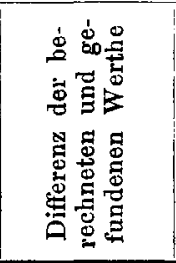 & 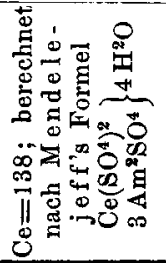 & 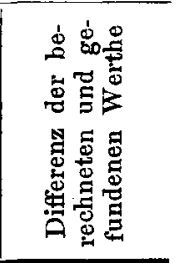 \\
\hline $\begin{array}{l}\mathrm{Am}^{2} \mathrm{O} \\
\mathrm{SO}^{3} \\
\mathrm{CeO}^{2} \\
\mathrm{H}^{2} \mathrm{O}\end{array}$ & $\begin{array}{r}19,17 \\
50,01 \\
21,85 \\
\left.8,97^{* *}\right)\end{array}$ & $\begin{array}{r}18,45 \\
50,47 \\
22,34 \\
8,52\end{array}$ & $\begin{array}{c}+0,72 \\
-0,46 \\
-0,49 \\
+0,20 \\
\text { Mit. }= \pm 0,47\end{array}$ & $\begin{array}{r}19,55 \\
50,13 \\
21,30 \\
\left.9,02^{* * *}\right)\end{array}$ & $\begin{array}{c}-0,38 \\
-0,12 \\
+0,55 \\
-0,05 \\
\text { Mit. }= \pm 0,27\end{array}$ \\
\hline
\end{tabular}

*) Nicht die Formel mit $3 \mathrm{H}^{2} \mathrm{O}$, von welcher $\mathrm{Rammelsberg}$ spricht (Berichte der deutschen chemischen Gesellschaft 6, 86).

**) Aus der Differenz berechnet.

***) Im Exsiccator über Schwefelsäure entweichen 6,7 pC. Wasser, d. h. genau $3 \mathrm{H}^{2} \mathrm{O}$ von den vorbandenen $4 \mathrm{H}^{2} \mathrm{O}$. 
Aus der angeführten Tabelle erhellt, dafs meine Formel nicht blofs einfacher ist, sondern auch um das Doppelte genauer der Analyse, als die von R ammelsberg, entspricht. Man wird in dieser Ueberzeugung noch dadurch bestärkt, dafs Ceroxydsalze im Allgemeinen von Wasser zersetzt werden, man daher in aus Wasser umkrystallisirtem Salze mehr $\mathrm{Ce}^{2}$ erwarten darf, als der Rechnung entspricht, und nicht weniger, wie von Rammelsberg im Vergleich mit seiner Formel gefunden worden ist. Desgleichen ist eher ein geringer Verlust bei der Ammoniakbestimmung zu erwarten und kein Ueberschufs, welchen Rammelsberg nach seiner Formel zuläfst; um so mehr, als jede von den drei angefülrten Analysen mehr Ammoniak ergeben hat, als Rammels berg's Formel entspricht. Mit einem Worte, das erwähnte Doppelsalz, welches R ammelsberg als Hauptstütze zur Widerlegung meiner Ausichten dient, spricht eher zu Gunsten von $\mathrm{CeO}^{2}$ als zu Gunsten eines hypothetischen Oxyds $\left(\mathrm{Ce}^{2} \mathrm{O}^{3}\right.$ bei $\mathrm{Ce}=92$ oder $\mathrm{Ce}^{4} 0^{9}$ wenn $\left.\mathrm{Ce}=138\right)$, dessen Existenz bei der früheren Anschauungsweise, die Rammelsberg noch gegenwärtig zu seiner Formel geführt hat, vorausgesetzt wurde. Ich bin überzeugt, dafs $R$ ammelsberg meine Formel, zu welcher er selbst bei richtiger Vorstellung über das Ceratomgewicht gelangt wäre, annehmen wird *).

Aufserdem ist kein Salz mit einem höheren Oxyde als $\mathrm{Ce}^{2}$ bekannt; folglich mufs das $0 x y d \mathrm{Ce}^{2} 0^{3}(\mathrm{Ce}=92)$, oder

*) Nimmt man für Ce das Atomgewicht 140 an, welches nach dem periodischen Gesetze besser als 138 ersterem entspricht (diese Annalen Suppl. 8, Tab. I und II, S. 187), so stimmt meine Formel noch mehr mit Rammelsberg's Analysen überein; die berechneten Werthe sind nämlich $19,50 \mathrm{Am}^{2} \mathrm{O}, 50,00 \mathrm{SO}^{3}, 21,50 \mathrm{CeO}^{2}$ und $9,00 \mathrm{H}^{2} \mathrm{O}$, was einen Mittelfehler von $\pm 0,18 \mathrm{pC}$. giebt. Ich setze voraus, dafs die Fähigkeit dieses Salzes, gut zu krystallisiren, ein geeignetes Mittel bieten wird, um reines Cerium darzustellen und dessen Atomgewicht zu bestimmen. 
$\mathrm{Ce}^{4} \mathrm{O}^{9}(\mathrm{Ce}=138)$, welches früher von Berzelius, Herrmann u. A. und gegenwärtig noch von $R$ ammelsberg angenommen worden ist, als fictives, nicht existirendes betrachtet werden. Somit gehören alle bekannten Cersalze zu folgenden drei Classen :

I. Oxydulsalze, $\mathrm{Ce}^{2} \mathrm{X}^{6}$ oder $\mathrm{CeX}^{3}$; sie sind farblos, wenn keine anderen gefärbten $0 x y d e$ zugegen sind; wirken nicht oxydirend, wenn sie keine Beimengung oxydirender Agentien enthalten; entsprechen dem Oxydul $\mathrm{Ce}^{2} \mathrm{O}^{3}$ (früher $\mathrm{CeO}$ ). Viele einfache $\left(\mathrm{Ce}^{2}\left[\mathrm{SO}^{4}\right]^{3}+4\right.$ bis $9 \mathrm{H}^{2} \mathrm{O}$; $\left.\mathrm{Ce}^{2}\left[\mathrm{NO}^{3}\right]^{6}, 12 \mathrm{H}^{2} \mathrm{O}\right)$, sowie Doppelsalze $\left(\mathrm{Ce}^{2} \mathrm{R}^{3}\left[\mathrm{NO}^{3}\right]^{12}, 24 \mathrm{H}^{2} \mathrm{O}^{*}\right)$, wo $\mathrm{R}=\mathrm{Zn}$, $\left.\mathrm{Mg}, \mathrm{Ni}, \mathrm{Mn} ; \mathrm{CeK}\left[\mathrm{SO}^{4}\right]^{2} \mathrm{H}^{2} \mathrm{O}^{* *}\right), \mathrm{CeK}^{3}\left[\mathrm{SO}^{4}\right]^{3 * * *}$ und andere) sind gut studirt und deren Zusammensetzung unzweifelhaft.

II. Oxyduloxydsalze, $\mathrm{Ce}^{2} \mathrm{X}^{6}, \mathrm{Ce}^{2} \mathrm{X}^{8}$, d. h. $\mathrm{Ce}^{2} \mathrm{X}^{7}$ und vielleicht noch $\mathrm{Ce}^{3} \mathrm{X}^{8}$; andere Formen sind nicht mit Sicherheit bekannt. Diese Salze bilden den Uebergang von der ersten zur dritten Classe und entstehen aus letzteren Salzen unter Sauerstoffentwickelung. Hierher gehört das oben erwähnte Salz $\mathrm{Ce}^{4}\left(\mathrm{SO}^{4}\right)^{7}, 24 \mathrm{H}^{2} \mathrm{O}$.

III. Oxydsalze, $\mathrm{Ce}^{2} \mathrm{X}^{8}$ oder $\mathrm{CeX}^{4}$, entsprechen dem Oxyde $\mathrm{CeO}^{2}$ (früher $\mathrm{Ce}^{3} \mathrm{O}^{4}$ ), sind gelb oder orangeroth gefärbt, unbeständig, gehen unter Zersetzung in Salze der zweiten Classe über; wirken oxydirend, wobei Salze der ersten Classe entstehen; werden oft von Wasser unter Bildung basischer Salze $\mathrm{Ce}^{2} \mathrm{O}^{3} \mathrm{X}^{2}$ und anderer, da $\mathrm{CeO}^{2}$ eine

*) $\mathrm{Zu}$ diesem Typus gehören ausgezeichnet krystallisirende Salze, welche in der Krystallisationsfahigkeit den Alaunen gleichkommen.

**) Die Zusammensetzung dieses Salzes gleich Aluun ohne Wasser; das entsprechende Tl- und Na-Salz enthält eben so viel Wasser, das Ammoniumsalz 3 bis 4 aq.

***) Dieses Salz entspricht dem vorhergehenden, in welchem 1 aq durch $\mathrm{K}^{2} \mathrm{SO}^{4}$ ersetzt ist, wie in dem Salze $\mathrm{ZnSO}^{4}, 7 \mathrm{H}^{2} \mathrm{O}$ ein $\mathrm{H}^{2} \mathrm{O}$ durch $\mathrm{K}^{2} \mathrm{SO}^{4}$ vertreten sein kann. 
schwache Basis ist, zersetzt. Viele Salze sind unzweifelhaft : das gelbe Salz von Herrmann und Rammelsberg $\mathrm{Ce}\left(\mathrm{SO}^{4}\right)^{2}, 4 \mathrm{H}^{2} \mathrm{O}$, das gelbe Ammoniumdoppelsalz $\mathrm{Ce}^{2}\left(\mathrm{SO}^{4}\right)^{4}$, $3 \mathrm{Am}^{2} \mathrm{SO}^{4}, \mathrm{n}$ aq. , das oben erwähnte leichter lösliche orangerothe Salz und andere.

Somit könnte die Frage über die complicirte Zusammensetzung der Cersalze, welche von früheren Forschern und mit ihnen von R ammelsberg angenommen wird, als gelöst betrachtet werden. $\mathrm{Zu}$ den oben erwähnten Typen gehören alle von $R$ ammelsberg $u$. A. studirten Cerverbindungen.

Nachdem Rammelsberg im gröfsten Theil seiner $\mathbf{A b}-$ handlung Betrachtungen über die Zusammensetzung der erwähnten Salze mitgetheilt hat, macht er am Ende derselben noch Bemerkungen zweifacher Art gegen das von mir abgeänderte Atomgewicht des Ceriums. Erstens soll gezeigt werden, dafs der von mir dem Cerium angewiesene Platz (in der achten Reihe, Gruppe IV des periodischen Systems) nicht dessen Analogieen entspricht. Diese Bemerkungen lassen voraussetzen, dafs $R$ ammelsberg im Allgemeinen mit dem System nach dem periodischen Gesetze einverstanden ist, was ich für viel wichtiger, als die einzelnen Details halte, und dafs nach Widerlegung meiner Ansicht dem Cerium im System ein mehr entsprechender Platz angewiesen werden würde; doch läfst Rammelsberg letzteren Punkt unbeantwortet und mit dem alten Atomgewicht findet Cerium im System keinen Platz (diese Annalen Suppl. 8). Folglich müssen alle folgenden Erwiderungen Rammelsberg's von vorn herein als unsicher erscheinen, was sich denn auch bei genauer Betrachtung derselben herausstellt. Rammelsberg bemerkt, dafs das Ceriumoxyd $\mathrm{CeO}^{2} *$ ), obgleich in eine Gruppe mit $\mathrm{TiO}^{2}, \mathrm{ZrO}^{2}$ gestellt, sich von denselben in so fern unter-

*) Vgl. diese Annalen Suppl. 8, 189. 
scheidet, als es freies Chlor aus Chlorwasserstoff entwickelt. Es läfst sich darauf erwidern, dafs auch in der Gruppe $\mathrm{Al}^{2} \mathrm{O}^{3}$, $\mathrm{In}^{2} \mathrm{O}^{3}, \mathrm{Tl}^{2} \mathrm{O}^{3}$, oder $\mathrm{SiO}^{2}, \mathrm{SnO}^{2}, \mathrm{PbO}^{2}$ blofs je ein Glied als Hyperoxyd reagirt. Bekannterweise wirkt $\mathrm{H}^{2} \mathrm{TeO}^{4}$ oxydirend auf Chlorwasserstoff ein; sollte denn darauf hin die Analogie zwischen $\mathrm{H}^{2} \mathrm{SO}^{4}$ und $\mathrm{H}^{2} \mathrm{TeO}^{4}$ verneint werden ? Eine Hauptstütze von der Richtigkeit meiner Meinung sehe ich in der folgerechten Aenderung der Eigenschaften in der Reihe :

$\mathrm{TiO}^{2}, \mathrm{ZrO}^{2}, \mathrm{CeO}^{2}, \mathrm{LaO}^{2}$, $\mathrm{ThO}^{2}$ (IV. Gruppe, paare Reihen).

Die zwei ersten Bioxyde sind Säuren, die zwei letzteren Basen, in der Mitte steht $\mathrm{CeO}^{2}$. Welche sind aber die Eigenschaften eines zwischen schwachen Säuren einerseits und schwachen Basen andererseits stehenden Oxyds? Jedermann wird antworten : die eines Hyperoxyds, Zersetzung von Chlorwasserstoff unter Chlorentwickelung. $\mathrm{TiO}^{2}, \mathrm{ZrO}^{2}$ sind als saure Oxyde auf Chlorwasserstoff ohne Einwirkung, während $\mathrm{LaO}^{2}$ und $\mathrm{ThO}^{2}$ Salze der einzigen Oxydationsstufe liefern. Folglich mufs $\mathrm{CeO}^{2}$ Chlor aus Chlorwasserstoff entwickeln, weil es eine wenig energische Base von grofsem Sauerstoffgehalt ist, und derselben eine niedere, beständigere $0 x y$ dationsstufe mit deutlicher basischen Eigenschaften entspricht. Dieses wird noch deutlicher, wenn wir annehmen, dafs $\mathrm{CeCl}^{4}$ sich bilde, dafs letztere Verbindung aber noch unbeständiger sei, als $\mathrm{CuCl}^{2}, \mathrm{TlCl}^{3}, \mathrm{PtCl}^{4}$, dabei aber wie diese eine beständigere, niedere Verbindung $\mathrm{CeCl}^{3}$, welche dem Oxydul entspricht, gebe; dem ähnlich entwickeln $\mathrm{Mn}^{2} \mathrm{O}^{3}$ und $\mathrm{MnO}^{2}$ Chlor aus Salzsäure, wobei $0 x y d u l s a l z ~ e n t s t e h t *$ ). Aufserdem weist Rammelsberg darauf hin, dafs $\mathrm{LaCl}^{4}$ nicht flüchtig sei, im Widerspruch mit den übrigen $\mathrm{RCl}^{4}$; dagegen genügt, daran

*) Es lärst sich ein beständigeres $\mathrm{CeF}^{4}$ voraussetzen, wio $\mathrm{MnF}^{4}$; dasselbe wird wahrscheinlich ähnliche Doppelsalze wie $\mathrm{Zr}$, Th liefern. 
zu erinnern, dafs $\mathrm{SiF}^{4}$ gasförmig ist, während die übrigen bekannten $\mathrm{RF}^{4}$, z. B. $\mathrm{ZFF}^{4}$, nicht flüchtig sind. Was die Bemerkung, dafs $\mathrm{LaO}^{2}, \mathrm{Ce}^{2} 0^{3}$ Salmiak zersetzen, was die übrigen Oxyde $\mathrm{R}^{2} \mathrm{O}^{3}, \mathrm{RO}^{2}$ nicht thun, anbelangt, so verweise ich zur Antwort auf die Geschichte des Berylliums und auf S. 172 dieser Annalen Suppl. 8.

Die Bemerkungen zweiter Art betreffen den Isomorphismus der Ceritmetalle. R a m m el s b e r g bringt wieder die Frage über den Isomorphismus des Didymoxyds mit Cadmiumoxyd und des Ceroxyds mit Magneteisenstein ( $\mathrm{Di}^{2} \mathrm{O}^{3}, \mathrm{CdO} ; \mathrm{CeO}^{2}, \mathrm{Fe}^{3} \mathrm{O}^{4}$ ) in $\mathrm{An}-$ regung und verneint daraufhin die Möglichkeit, dem Didymoxyd die Formel $\mathrm{Di}^{2} \mathrm{O}^{3}$, sowie dem Ceroxyd $\mathrm{CeO}^{2}$ zu geben, oder fordert entgegengesetzten Falls für Zinnober die Formel $\mathrm{Hg}^{2} \mathrm{~S}^{3}$. Dagegen genügt, an von Vielen und auch von $R$ a m mels berg beobachtete Thatsachen zu erinnern. Noch vor einem Jahre hat er selbst (Berichte der deutschen chemischen Gesellschaft 5, 18) den Isomorphismus von $\mathrm{FeNb}^{2} \mathrm{O}^{6}, \mathrm{FeWO}^{4}, \mathrm{Ta}^{2} \mathrm{O}^{5}, \mathrm{WO}^{3}, \mathrm{TiO}^{2}$ hervorgehoben und es ist zu verwundern, dafs er nicht damals abgeänderte Atomgewichte für die angeführten Elemente, sowie für deren Analoge verlangt hat. Ueber den Isomorphismus verschieden constituirter Verbindungen sind schon früher von Laurent, Dana, Marignac Beispiele gegeben. Ich will nur an den Isomorphismus von $\mathrm{ZnO}$ und $\mathrm{Al}^{2} \mathrm{O}^{3}, \mathrm{CaCO}^{3}, \mathrm{KNO}^{3}$ und $\mathrm{K}^{2} \mathrm{ZrF}^{6}$ (M arignac, Ann. chim. phys. [3] 60), oder von $\mathrm{R}^{2} \mathrm{ZrF}^{6}, \mathrm{R}^{2} \mathrm{NbOF}^{5}$ und $\mathrm{R}^{2} \mathrm{~W} 0^{2} \mathrm{~F}^{4}$ erinnern. Die bis jetzt bekannten Thatsachen zeigen schon, dafs Isomorphismus entweder bei vollkommen gleichartiger Zusammensetzung auftritt, oder bei gleicher Atomenanzahl und ähnlicher chemischer Form sowie übereinstimmenden Eigenschaften der in den Verbindungen enthaltenen Elemente, oder bei einiger entfernter Aehnlichkeit oder Aequivalenz, jedoch beständig bei aus analogen Elementen bestehenden Verbindungen. Giebt man dem Ceroxyd die Formel $\mathrm{Ce}^{2} 0^{4}$, so stellt sich einige 
Aehnlichkeit mit dem Magneteisenstein heraus *). Dieselbe reguläre Krystallform besitzten auch $\mathrm{Cu}^{2} \mathrm{O}, \mathrm{MgO}$, $\mathrm{NiO}$; dennoch liegt kein Grund vor, letzteren Oxyden die Formel des Magneteisensteins zu geben. Vergleicht man die Formeln $\mathrm{Di}^{2}\left(\mathrm{SO}^{4}\right)^{3}$, $8 \mathrm{H}^{2} \mathrm{O}, \mathrm{Cd}^{3}\left(\mathrm{SO}^{4}\right)^{3}, 8 \mathrm{H}^{2} \mathrm{O}$, so sehen wir dieselbe Aehnlichkeit, wie zwischen $\mathrm{Ce}^{2} \mathrm{O}^{4}$ und $\mathrm{Fe}^{3} \mathrm{O}^{4}$. Im rhomboëdrischen System ist bei $\mathrm{Al}^{2} \mathrm{O}^{3}$ die Endfläche zur Pyramide unter einem Winkel von $118^{0}, 49^{\prime}$ geneigt, bei $\mathrm{ZnO}$ unter $118^{\circ}, \boldsymbol{7}^{\prime}$; trotzdem hat noch Niemand an den Formeln dieser 0xyde gezweifelt. Wer aber wird behaupten, dafs das Ceroxyd in der That das Moleculargewicht $\mathrm{CeO}^{2}$ und nicht $\mathrm{Ce}^{\mathrm{n}} \mathrm{O}^{2^{\mathrm{n}}}$ besitze? In den isomorphen Salzen $\mathrm{CaCO}^{3}$ und $\mathrm{K}^{2} \mathrm{ZrF}^{6}$ sind $\mathrm{Ca}-\mathrm{K}^{2}$ und $0^{3}-\mathrm{F}^{6}$ einander äquivalent; weshalb sollte denn zwischen den äquivalenten Mengen $\mathrm{Di}^{2}-\mathrm{Cd}^{3}$, welche $\mathrm{H}^{6}$ entsprechen, nicht Isomorphismus herrschen können? $\mathrm{Ce}^{2}$ in $\mathrm{CeO}^{2}$ ist $\mathrm{Fe}^{3}$ in $\mathrm{Fe}^{3} 0^{4}$ auch äquivalent. Aufserdem ist die reguläre Krystallform am Ceriumoxyd nicht genügend genau nachgewiesen ( $N$ ordenskiöld), die Zusammensetzung des schwefelsauren Didymoxyds aber hat bis auf Marignac's letzte Untersuchung (Archiv de Sc. phys. et nat. 1873, Mars) zu Zweifeln Anlafs gegeben (siehe Zschiesche, Journal für pract. Chemie 10\%, 77). Gegenwärtig ist gezeigt, dafs die Cer- und Didymsalze mit $8 / 3$ aq. nicht isomorph sind, dagegen aber die Cer- und Lanthansalze mit 3 aq. Weiter behauptet $R$ am melsberg, "die Isomorphie der drei Ceritmetalle sei aufser Frage". Dem war bis auf die oben erwähnte Untersuchung von Marignac nicht so; im Gegentheil war diese Isomorphie noch kein Mal genügend bewiesen worden. Es gab früher wohl einen Grund, dieselbe anzunehmen; aber gegenwärtig hat letzterer seine Bedeutung verloren. Es war

*) Dieses Verhältnifs entspricht dem Verbältnifs von $\mathrm{TiO}^{2}$ zu $\mathrm{WO}^{3}$, oder $\mathrm{Ti}^{3} \mathrm{O}^{6} \mathrm{zu} \mathrm{W}^{2} \mathrm{O}^{6}$, oder des Wolframs zum Columbit. 
nämlich beobachtet worden (Bunsen, Holtzmann, Rammelsberg), dafs die salpetersauren Doppelsalze der Ceritmetalle mit $\mathrm{Mg}$ und anderen durch Krystallisirenlassen nicht getrennt werden können. Zugleich wurde angenommen, dafs in denselben Ceroxyd und Oxydul enthalten sei. Nun fragt sich, mit welchen von beiden Oxyden Didym und Lanthanoxyd isomorph seien? Zschiesche hat nachgewiesen, dafs die erwähnten Doppelsalze Oxydulsalze (v. Lang) mit einer Beimengung von Oxydsalzen seien. Gegenwärtig spricht sich Rammelsberg (a. a. 0.) auch dahin aus; man könnte weiter voraussetzen, dafs in den Salzen auch Beimengungen von Di und La enthalten seien. Ich kann denken, dafs Didym- und Lanthanoxyd zum wenigsten einander nicht vollkommen isomorph seien, weil ich durch Umkrystallisiren des salpetersauren Lanthanammondoppelsalzes Lanthan von Didym habe trennen können; das Didym bleibt in den Multerlaugen. Dasselbe findet statt, wenn man nach Mosander La und Di als schwefelsaure Salze trennt. Wenn sich dabei in einem bestimmten Momente Salze, welche Di und La gleichzeitig enthalten, bilden, so haben wir oben schon eine analoge Erschei nung angeführt, nämlich gemischte Ceroxydul-Ceroxydsalze. Ich nehme an, dafs Didyınoxyd (oder Lanthanoxyd) $\mathrm{Ce}^{2} 0^{3}$ analog zusammengesetzt sei, dagegen Lanthanoxyd (oder Didymoxyd) $\mathrm{Ce}^{2}$ entspreche. Wenn man daher gemischte Ceroxydulceroxydsalze zuläfst, mufs man auch gemischte Salze von Didymund Lanthanoxyd für möglich halten *). Die letzten oben er-

*) Bezüglich der Frage über Isomorphismus dürfen bei unseren gegenwärtigen Kenntnissen die gewichtigen und geistreichen $\mathrm{Be}-$ trachtungen Laurent's (Méthodes de Chimie) nicht unbemerkt bleiben. Marignac's Untersuchungen über die Fluordoppelsalze, sowic Wyroubow's über die Ferridcyansalze geben die Richtung, in welcher derartige Fragen weiter auszuarbeiten sind, an. Solche Arbeiten sind im Interesse der Molecularmechanik fester chemischer Verbindungen sehr zu wünschen. 
wähnten Untersuchungen Marignac's, welche nach Rammelsberg's Abhandlung erschienen sind, geben Grund zu behaupten, dafs Ceroxydul mit Lanthan- und Didymoxyd in der That isomorph seien, d. h. dafs die entsprechenden Salze derselben, besonders die complicirten Doppelverbindungen von äquivalenter Zusammensetzung sind und in übereinstimmenden Krystallformen oft auftreten, wenn auch in den einfacheren Salzen nicht immer Isomorphismus stattfindet. Leider hat Marignac nicht versucht, gemischte Krystalle zu erhalten, welche die einzig sichere Garantie von vollständigem Isomorphismus bieten. Der Umstand, dafs die entsprechenden Salze gleiche Mengen Krystallwasser und anderer Salze $\left(\mathrm{PtCl}^{4}\right.$, $\mathrm{NH}^{4} \mathrm{NO}^{3}$ ) binden, beweist noch nicht völligen Isomorphismus; denn in dieser Beziehung können Salze sehr verschieden zusammengesetzter Oxyde einander gleichen. So enthalten z. B. die Nitrate $\mathrm{Al}\left(\mathrm{NO}^{3}\right)^{3}, \mathrm{Cr}\left(\mathrm{NO}^{3}\right)^{3}, \mathrm{Fe}\left(\mathrm{NO}^{3}\right)^{3}$, ebenso wie $\mathrm{Mn}\left(\mathrm{NO}^{3}\right)^{2}$, $\operatorname{Mg}\left(\mathrm{NO}^{3}\right)^{2}, \mathrm{Co}\left(\mathrm{NO}^{3}\right)^{2}, \mathrm{UrO}^{2}\left(\mathrm{NO}^{3}\right)^{2}$ äquivalente Mengen Wasser, nämlich $3 \mathrm{H}^{2} \mathrm{O}$ auf je ein $\mathrm{NO}^{3}$. Gegenwärtig ist es unbekannt, ob dabei die Krystallform erhalten bleibt oder nicht; einiger Grund ist vorhanden, anzunehmen, dafs Isomorphismus stattfinden kanı bei verschiedener Zusammensetzung der Oxyde und gleichem Krystallwassergehalt, da, wie oben gezeigt worden ist, verschieden zusammengesetzte, aber einander äquivalente Mengen oft isomorph sind.

Der Isomorphismus mufs voraussetzlich nicht nur durch äquivalente Zusammensetzung, sondern auch durch den chemischen Charakter der enthaltenen Oxyde bedingt sein. So können entsprechende Salze von $\mathrm{ZnO}$ und $\mathrm{Al}^{2} \mathrm{O}^{3}$ einander isomorph sein, weil letztere Oxyde obgleich von ungleicher Zusammensetzung ähnliches chemisches Verhalten in ihren Reactionen zeigen. Dasselbe Verhältnifs tritt bei Ceroxydul, Didym- und Lanthanoxyd auf; deshalb ist auch bei letzteren trotz ungleicher Zusammensetzung der Oxyde Isomorphismus 
denkbar. Aufserdem werden in den erwähnten Untersuchungen Marignac's neue Thatsachen bezüglich der Zusammensetzung von Doppelsalzen mitgetheilt, welche zeigen, dafs die Ceritmetalle Verbindungen anderer Art, als die übrigen bekannten Oxyde RO liefern; so enthalten z. B. die Platindoppelsalze $3 \mathrm{PICl}^{4}$ auf $4 \mathrm{RCl} 36$ aq. und nicht $\mathrm{PtCl}^{4} \mathrm{RCl}^{2} 6$ bis 8 aq. Diese Thatsachen werden erst dann in rechten Lichte aufgefafst werden können, wenn die Zusammensetzung der Platindoppelsalze solcher Oxyde, wie $\mathrm{Al}^{2} \mathrm{O}^{3}, \mathrm{Fe}^{2} \mathrm{O}^{3}, \mathrm{ZrO}^{2}$ und dergleichen mehr bekannt sein wird. Um in den Ceritoxyden eine besondere, selbstständige Classe deutlicher, anders als R0 zusammengesetzter Basen zu erblicken, genügt es, daran zu erinnern, dafs alle drei Ceritmetalle mit Alkalinitraten leicht Doppelsalze geben, wozu, wie ich bei unter den verschiedensten Bedingungen ausgeführten Versuchen mich zu überzeugen Gelegenheit gehabt habe, weder $\mathrm{Mg} 0$, noch $\mathrm{Al}^{2} 0^{3}, \mathrm{CoO}, \mathrm{Zn0}{ }^{*}$ ) befähigt sind. Bis jetzt sind blofs schwach-basische Oxyde von den Formen $\mathrm{R}^{2} \mathrm{O}^{3}$ und $\mathrm{RO}^{2}$ gut bekannt; giebt man aber den Ceritmetallen in Uebereinstimmung mit dem periodischen Gesetze die von mir vorgeschlagenun Atomgewichte, so stehen für erstere bei den angeführten Oxydformeln deutliche Basen zu erwarten. Von besonderem Interesse wäre in dieser Beziehung ein vergleichendes Studium von $\mathrm{ThO}^{2}$ und von den Oxyden $\operatorname{In}^{2} 0^{3}, \mathrm{Tl}^{2} \mathrm{O}^{3}$ als einzige bekannte, deutlich-basische, höhere Oxyde, deren Isomorphismus nicht studirt worden ist.

Ich mufs zugleich bemerken, dafs das periodische System, indem es dem Cerium einen bestimmten Platz anweist, in Bezug auf Lanthan und Didym zu einigen $Z_{w}$ weifeln Anlafs giebt. Mit anderen Worten, es stellt die Zusammensetzung der Oxyde $\mathrm{Ce}^{2} 0^{3}, \mathrm{CeO}^{2}$ aufser $\mathrm{Zw}$ eifel und macht den Umstand sehr wahrscheinlich, dafs La- und Di-Oxyd wie $\mathrm{Ce}^{2} \mathrm{O}^{3}$ und $\mathrm{CeO}^{2}$

*) $\mathrm{Blos} \mathrm{TiO}^{2}$ und $\mathrm{UrO}^{3}$ hat mir ein solches Doppelsalz gegeben. 
zusammengesetzt seien; doch läfst sich nicht mit Gewifsheit behaupten, welches $0 x y d \mathrm{Ce}^{2} \mathrm{O}^{3}$ entspreche und welches $\mathrm{CeO}^{2}$ analog zusammengesetzt sei, weil die Bestimmungen der Aequivalentgewichte von Di und La zu Zweifeln führen, welche blofs durch weitere Untersuchungen zu beseitigen sind.

Auf Grund aller dargelegten Betrachtungen mufs ich Rammelsberg's Erwiderungen ihre Bedeutung absprechen; ich glaube, dafs die von Ihm gelieferten Thatsachen eher zu Gunsten einer Aenderung in dem Atomgewicht von Cerium sprechen, als letztere widerlegen. Somit komme ich zu nachstehenden Schlufsfolgerungen :

1) Die periodische Gesetzmäfsigkeit führt zu Aenderungen in den Atomgewichten von $\mathrm{Ce}, \mathrm{Di}, \mathrm{La}$, so dafs die Sauerstoffverbindungen dieser Metalle folgende Zusammensetzung haben : $\mathrm{Ce}^{2} \mathrm{O}^{3}, \mathrm{CeO}^{2}, \mathrm{Di}^{2} \mathrm{O}^{3}(?), \mathrm{LaO}^{2}(?)$.

2) Die von $R$ a m m els berg ausgeführten Analysen lassen sich bei Annahme der neuen Ceroxydformel $\mathrm{CeO}^{2}$ durch genauere Formeln, als nach der alten Vorstellung wieder geben.

3) Die nach der alten Vorstellung möglichen Ceroxyde, welche mehr Sauerstoff, als $\mathrm{CeO}^{2}$ enthalten, existiren nicht, z. B. $\mathrm{Ce}^{2} 0^{3}(\mathrm{Ce}=92)$, welche $\mathrm{Ce}^{4} 0^{9}$ entspricht, wenn $\mathrm{Ce}=138$.

4) Die neuen Atomgewichte, $\mathrm{Ce}=140$ (138?), $\mathrm{Di}=$ $138 ?$ und $L a=180$ ? entsprechen den Analogieen dieser Metalle und bieten die Möglichkeit, die oxydirenden Eigenschaften von $\mathrm{CeO}^{2}$ zu erklären.

5) Da Fälle von Isomorphismus an ähnlichen aus einer ungleichen Anzahl Atomen bestehenden Körpern (z. B. ZnO und $\mathrm{Al}^{2} \mathrm{O}^{3}, \mathrm{CaCO}^{3}$ und $\mathrm{K}^{2} \mathrm{ZrF}^{6}, \mathrm{FeNb}^{2} \mathrm{O}^{6}$ und $\mathrm{FeWO}^{4}, \mathrm{NH}^{4} \mathrm{X}$ und $\mathrm{KX}$ ) bekannt sind, so widerspricht der Isomorphismus von $\mathrm{Ce}^{2} \mathrm{O}^{4}$ und $\mathrm{Fe}^{3} \mathrm{O}^{4}$, oder $\mathrm{Di}^{2}\left(\mathrm{SO}^{4}\right)^{3}, 8 \mathrm{H}^{2} \mathrm{O}$ und $\mathrm{Cd}^{3}\left(\mathrm{SO}^{4}\right)^{3}$, $8 \mathrm{H}^{2} \mathrm{O}$ nicht den von mir angenommenen Atomgewichten der Ceritmetalle. 
6) Da die Zusammensetzung der Doppelsalze der Ceritmetalle Eigenthümlichkeiten zeigt und keine gemischten Salze derselben untereinander und mit $\mathrm{RO}$-Salzen beobachtet worden sind, so ist deren Isomorphismus mit RO zweifelhaft.

7) Da weder die von mir bestimmte Wärmecapacität des Ceriums, noch die atomanalogen Verhältnisse der Ceroxyde, noch die Zusammensetzung der Cersalze mit dem neuen Atomgewicht von Cerium in Widerspruch stehen; da mit dem neuen Atomgewicht alle dieses Metall betreffenden Thatsachen genauer, einfacher und ohne Annahme einer besonderen hypothetischen 0xydationsform aufgefafst werden können; da schliefslich das neue Atomgewicht von Cerium durch das von mir bemerkte periodische Gesetz, an dessen Genauigkeit kein Grund zu zweifeln vorliegt, hervorgerufen ist : so behaupte ich, dafs Rammelsberg keinen Grund gehabt hat, die von mir vorgeschlagenen Aenderungen an den Atomgewichten der Ceritmetalle für nicht annehmbar zu erklären. Man kann gegenwärtig mit vollem Rechte behaupten, dafs das alte Atomgewicht des Ceriums durch das neue, von dem periodischen Gesetze vorausgesehene ersetzt werden mufs. Aufserdem behaupte ich, dafs man gegenwärtig keine Betrachtungen über Elemente anstellen darf und kann, ohne das periodische Gesetz zu beachten, eben so wie man bei Erörterungen über zusammengesetzte Körper die Gesetze vom Molecul, von den Substitutionen und von den Grenzen nicht umgehen kann. Diese Grundthesis hat mich denn auch bewogen, Rammels berg's Erwiderungen einer eingehenderen Analyse zu unterwerfen.

St. Petersburg, März 1873. 